\title{
REFLEXIÓN EN TORNO A LA APLICACIÓN DE HERRAMIENTAS PARA OPERAR SOBRE LAS LENGUAS Y EL CASO DE LA LENGUA DE SEÑAS URUGUAYA (LSU)
}

\author{
Leonardo Peluso Crespi*
}

\begin{abstract}
RESUMEN
Existen básicamente cuatro herramientas que se han aplicado a las lenguas con el fin de operar sobre ellas, si bien se podrían considerar algunas más que estas cuatro según se adopte un criterio más o menos amplio del concepto de herramienta. Estas herramientas son: los sistemas de escritura y sus diferentes soportes de materialización; las herramientas de registro o grabación; las herramientas de gramatización; y las tecnologías de la información y la comunicación. En este trabajo, consideraré la situación de todas estas herramientas, pero me detendré particularmente en los sistemas de escritura, dado que es la herramienta central que la humanidad ha aplicado sobre las lenguas y que impone estructuras y funciones sobre las otras.
\end{abstract}

Palabras clave: Sistemas de escritura. Herramientas de registro. Instrumentos de Gramatización. Lenguas de Señas.

\begin{abstract}
There are four basic tools that have been applied to languages in order to operate on them, although, depending on the concept of tool adopted, some more could be mentioned. These tools are: writing systems, recording devices (audio or video recorders), grammatization instruments (grammars and dictionaries), and technologies of information and communication. In this paper, I will consider the situation of these tools and their application to sign languages, specifically Uruguayan Sign Language (LSU). I will focus particularly on writing systems, since they are the basic tool that humanity has applied to languages, and that, in turn, imposes structures and functions on the other tools.
\end{abstract}

Keywords: Writing systems. Recording devices. Grammatization instruments. Sign Languages

* Magister por la Facultad de Humanidades y Ciencias de la Educación, Universidad de la República (FHCE/UdelaR). Profesor e coordinador de la Tecnicatura en Interpretación LSU - español, Facultad de Humanidades y Ciencias de la Educación, Universidad de la República (FHCE/UdelaR).E-mail: leonardo@psico.edu.uy 


\section{Los sistemas de escritura y transcripción}

Es probable que la primera herramienta se haya aplicado sobre las lenguas orales sea la escritura. La misma está compuesta por un sistema de grafemas, leyes ortográficas para su combinación y un soporte físico para su materialización.

\section{Las lenguas orales y los sistemas de escritura y de transcripción}

Los sistemas de escritura, según Sampson (1997), son conjuntos de marcas permanentes y visibles que permiten representar las diversas unidades de la lengua. Los diversos sistemas de escritura fueron agrupados por Sampson (1997) de acuerdo con el tipo de unidad que representan. Así, para este autor, la escritura semasiográfica se distingue de la glotográfica en que, en el primer tipo, se estarían representando ideas más o menos específicas, pero no enunciados de la lengua. Por ejemplo, sería el caso de los símbolos que aparecen en las etiquetas de la ropa en los que se indica acerca de cómo se debe lavar, planchar, etc. En estos signos no se representan enunciados, sino ideas específicas que, dado su convencionalización, pueden ser interpretados por casi cualquier ser humano integrado a la cultura global planetaria. Como no se representan enunciados de la lengua, para Sampson no serían sistemas de escritura en un sentido estricto. Por su parte, las escrituras glotográficas serían aquellas en las que sí se representan enunciados de la lengua a través de la representación de sus unidades.

Teniendo en cuenta esta distinción primaria, Sampson (1997) realiza luego una diferenciación entre los tipos de escritura glotográfica, utilizando la noción de doble articulación propuesta por Martinet (1966), quien distingue entre dos grandes tipos de unidades de la lengua: las unidades del segundo nivel de articulación, que se caracterizan por carecer de significado; y las unidades del primer nivel de articulación, que se caracterizan por tener significado. A partir de esto, Sampson separa los sistemas logográficos de aquellos fonográficos. Los sistemas logográficos son los que representan unidades del primer nivel de articulación, es decir, los que representan los morfemas de la lengua. Un claro ejemplo de este tipo de escritura son los Kanji, representativos de la cultura letrada oriental. Sampson plantea que teóricamente podrían existir sistemas de escritura que representen unidades 
polimorfémicas (por ejemplo palabras y no morfemas), sin embargo no se encuentran en la actualidad sistemas de este tipo dado que son muy poco económicos. Los sistemas fonográficos, en cambio, representan unidades del segundo nivel de articulación. Para Sampson habría tres tipos de sistemas de escritura fonográfica: los silábicos, aquellos que representan sílabas de la lengua (por ejemplo, algunos sistemas utilizados por los japoneses); los segmentales, que representan fonemas de las lenguas (por ejemplo, los sistemas de escritura utilizados en occidente); y los rasgales, aquellos que introducen información fonética (por ejemplo, según Sampson el sistema de taquigrafía Pitman).

Por su parte Sampson aclara que no existen sistemas puros, ya que en los diferentes sistemas de escritura que actualmente existen se encuentran trazos de otros sistemas.Esta mezcla ha aumentado exponencialmente en la actualidad con el advenimiento de nuevas tecnologías ( $\mathrm{sms}$, chats) que han llevado a la escritura a cumplir nuevas funciones, más relacionadas con la oralidad.

En independencia de los sistemas de escritura, la lingüística ha desarrollado tanto un sistema de transcripción fonográfico, que se coloca entre / /, como un sistema de transcripción fonética, el IPA, que se coloca entre [ ]. El primero se relacionaría con los sistemas fonográficos segmentales que propone Sampson y el segundo con los fonográficos rasgales. Ambos son especialmente útiles para el trabajo lingüístico con corpus transcripto, pero no se ha extendido su uso a los hablantes letrados de las lenguas. Por eso no se podrían llamar sistemas de escritura, estrictamente hablando, sino sistemas de transcripción.

\section{Las lenguas de señas y los sistemas de transcripción y de escritura}

En Uruguay la lengua de señas (LSU) no tiene escritura por el momento. Las comunidades sordas en esta región no han adoptado un sistema de escritura o notación para su lengua. Esto no quiere decir que no existan sistemas de escritura y de transcripción propuestos para representar a la lengua de señas. Sin embargo, los mismos no han tenido aceptación generalizada por parte del mundo sordo, siendo más bien artefactos útiles para investigadores sordos y oyentes que trabajan con las lenguas de señas (educadores, traductores, lingüistas) quienes necesitan poder trascribir la 
lengua para su estudio y manipulación. En este sentido podemos mencionar varios sistemas, que se han inventado con objetivos diferentes. Por un lado estarían los sistemas de transcripción: la mimografía de Bébian, el sistema de notación de Stokoe, el sistema de Liddell y Johnson, el HamNoSys, las glosas y el sistema TRELSU. Por otro lado estarían los sistemas de escritura: el signwriting y el sistema semasiográfico de los Testigos de Jehová.

En el caso de los sistemas de transcripción, si se sigue un orden cronológico se podría decir que el primer sistema de notación que aparece es la mimografía (mimographie) de Auguste Bébian, año 1825. Esta mimografía tenía un propósito educativo pero pretendía ser una escritura. En este sentido parece interesante señalar la conciencia que tenía Bébian, muy avanzada para su época, de la necesidad de que en la educación todas las lenguas participantes tengan un sistema de escritura.

Tal como se puede leer en Oviedo (2009) el sistema de notación de Bébian está compuesto por aproximadamente 190 caracteres a través de los cuales se representaban básicamente tres aspectos de las señas: el órgano articulador, que puede ser activo (la mano) o pasivo (una parte de la cabeza o del cuerpo); el movimiento del que se indica tanto su naturaleza (lineal, curvo, etc.) como su modalidad (velocidad, ritmo, etc.); y la expresión facial.

En síntesis, la mimografía es un sistema fonético en la medida que se transcriben los diferentes aspectos que conforman la seña (que posteriormente Stokoe (1960) denominará parámetros y que se corresponden con los rasgos distintivos de las lenguas orales) por lo que nunca funcionaría como sistema de escritura: los hablantes no podrían procesar la lengua a través de éste como se procesa la lengua a través de un sistema de escritura. Es un sistema simultáneo, dado que no muestra la articulación interna de la seña. Por otra parte es un sistema que no está plenamente formalizado ya que algunos de los aspectos de las señas no queda exhaustivamente sistematizados en este sistema. Ninguna comunidad educativa o científica ha adoptado este sistema para la transcripción de la lengua de señas.

Más de cien años después, y debido, en parte, al oscurantismo que recayó sobre las lenguas de señas y la educación de los sordos en el mundo occidental, recién en 1960 aparece el sistema de notación simultáneo que propone William Stokoe para la Lengua de Señas Americana (ASL). Para Oviedo (2009) el sistema de Stokoe está francamente influido en el de Bébian, si bien Stokoe nunca lo hace explícito. 
Este sistema de Stokoe (1960) no tenía una pretensión educativa en su propuesta, sino centradamente lingüística, por lo que tuvo un diferente impacto en la comunidad científica, siendo considerado el precursor de la lingüística de la lengua de señas.

Al igual que la mimografía, el sistema de notación de Stokoe es también un sistema de índole fonético, pero presenta sus diferencias importantes. Este sistema propone notar tres aspectos de las señas, que se denominan parámetros: Tab (ubicación espacial de la seña); Sig (movimiento) y Dez (configuración de la mano). Se elimina el aspecto expresión facial de la notación y se agrega de forma explícita el parámetro movimiento. La eliminación que hace Stokoe de la expresión facial de la notación, según mi perspectiva, ha tenido tan fuerte impacto en la lingüística de la lengua de señas que aún hoy es un aspecto de la seña que cuenta con muy poco desarrollo descriptivo, si bien para los propios sordos es uno de los aspectos centrales de su lengua.

En el sistema de Stokoe no se utilizan signos icónicos, sino que se pretende usar los signos de la máquina de escribir para la notación.

En síntesis, creo que si bien la pretensión de ambos sistemas era de tipo segmental (siguiendo la terminología de Sampson), por la propia complejidad que presentaban y porque, de hecho, lo que hacían era transcribir algunos de los rasgos distintivos (parámetros) de las lenguas de señas, terminaron siendo sistemas de tipo rasgal (es decir, fonéticos), haciendo realmente difícil su uso y más difícil aún su expansión como sistema de transcripción por parte de la comunidad sorda.

Posteriormente en los años ochenta aparece el modelo secuencial de Liddell (1984) y posteriormente de Liddell y Johnson (1986), con un claro objetivo lingüístico, en el que se proponía realizar una descripción fonética de las mismas. No tienen por propósito la escritura, sino la descripción fonética de las lenguas de señas.

Este nuevo modelo de transcripción ya se basa en los cinco parámetros, que se usan en la actualidad: configuración, movimiento, orientación, ubicación y rasgos no manuales. Se diferencia del modelo de Stokoe no solo en que ya se incluyen dos nuevos parámetros, sino también en el hecho revolucionario de romper con la simultaneidad que aparecía en los modelos anteriores. Se describe así una matriz segmental articulatoria en cada seña, según la cual la seña típica tendría tres momentos: detención, movimiento, 
detención. En cada detención co-ocurren configuración, orientación y ubicación. El movimiento está compuesto, a su vez, por varios aspectos, tales como tipo de movimiento e intensidad. Los rasgos no manuales, a su vez, ocurren durante toda la seña. Este modelo mostraba la articulación interna a la seña y pretendía reconocer el nivel fonológico y la doble articulación en las lenguas de señas. Para el caso de la LSU, Fojo y Massone (2012, p. 99) presentan una adaptación de este modelo:

\section{QUADRO 1}

\section{Transcripción XII}

\begin{tabular}{|c|c|c|c|c|c|}
\hline \multicolumn{6}{|c|}{ DESPEGAR-AVIÓN } \\
\hline \multicolumn{6}{|l|}{ MP } \\
\hline \multicolumn{6}{|c|}{ D MDMD } \\
\hline $\mathrm{CM}$ & $4+123-a+$ & \multirow{5}{*}{ Lin } & & \multirow{5}{*}{$\operatorname{lin}$} & \\
\hline UB & $\begin{array}{l}\text { M } \\
\text { próx } \\
\text { enfr } \\
\text { ip-TR }\end{array}$ & & $\begin{array}{l}\text { med } \\
\text { enfr } \\
\text { ip-TR }\end{array}$ & & $\begin{array}{l}\text { dist } \\
\mathrm{HO}\end{array}$ \\
\hline DI & $\begin{array}{l}\text { B-M } \\
\text { PS }\end{array}$ & & & & \\
\hline OR & $\begin{array}{l}\text { Int-M } \\
\text { PH }\end{array}$ & & & & \\
\hline RNM & & & & & \\
\hline
\end{tabular}

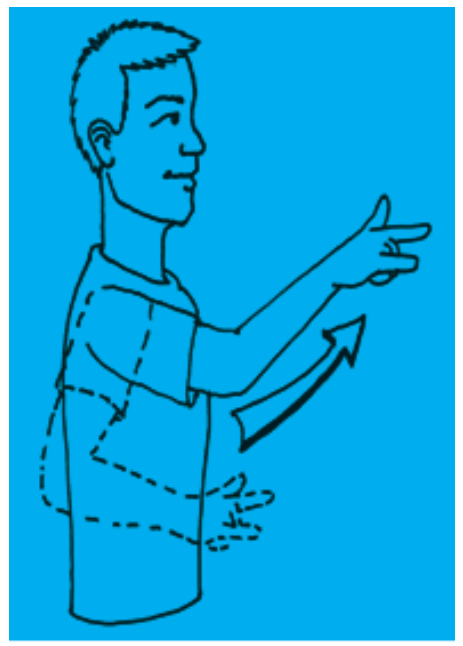

Se especifican los diferentes momentos articulatorios al interior de la seña (D: detención y M: movimiento) y luego los diferentes parámetros (CM: configuración de la mano; UB: ubicación; DI: dirección; OR: orientación y RNM: rasgos no manuales).

Este sistema tiene por objetivo la transcripción fonética de todas las lenguas de señas y no pretende constituirse en un sistema de escritura.

Al igual que los sistemas anteriormente descriptos, el HamNoSys es un sistema de transcripción fonética que aparece en los años noventa. En ese sentido sería similar al sistema IPA ya mencionado anteriormente. Por su poca, o nula, incidencia en estas latitudes, no me detendré en su descripción. 
Las glosas, por su parte, son usadas por los lingüistas para transcribir los enunciados realizados en lengua de señas con el fin de realizar su análisis. Se utilizan las palabras en mayúscula de la lengua oral para representar un significado aproximado de cada seña enunciada (o más de una palabra si así se requiere, dado que en muchos casos no hay correspondencia seña palabra) y se agrega información morfosintácitca y pragmática. De esa forma, si bien se utiliza la escritura de una lengua oral para representar la lengua de señas, se obtiene una representación del enunciado bastante ajustada a la morfosintaxis de la lengua de señas, con la inclusión de aspectos pargamáticos.

Presento tres ejemplos utilizados por Fojo y Massone (2012) para la transcripción de oraciones de la LSU:

neg

(19) CULPA PRO1-pos NO. PROBLEMA PRO3-pos.

"No es mi culpa. El problema es suyo" (Fojo y Massone, 2013: 113)

(24) DET NIÑO HIJO PRO2-pos.

int

“¿Ese niño es hijo tuyo?” (Fojo y Massone, 2012: 116)

El significado de la seña se escribe en mayúscula y cuando se necesita más de una palabra para dar ese significado, las mismas se unen con guiones. PRO1, PRO2 y PRO3 indican pronombres de primera segunda y tercera persona del singular. En caso de plural, se indica agregando - pl (ej. PRO1-pl) y en caso de pronombre posesivo se agrega -pos (ej. PRO2-pos). DET significa determinante. El orden de las glosas respeta el orden sintáctico de la LSU. La línea superior muestra el uso de rasgos no manuales que están en función sintáctica (int: interrogación; neg: negación; duda, etc.).

Por último, el descriptor TRELSU que se está elaborando en el Uruguay, se ubica en el nivel fonológico y propone seguir en la línea teórica planteada por Liddell y Johnson (1986) y luego reformulada por Massone (1993) para Argentina y Oviedo (2001) para la descripción de la lengua de señas colombiana, pero se diferencia en que en lugar de sostener la descripción en la escritura de una lengua oral, lo hace mediante el uso de un código creado ad hocpor el equipo de investigación, en el que se utilizan símbolos matemáticos, diseños geométricos y algunos signos del signwriting (BO- 
NILLA; PELUSO, 2010). Se describe, con estos lenguajes, la articulación interna de cada seña y los diferentes parámetros que están coimplicados, a modo de rasgos distintivos:

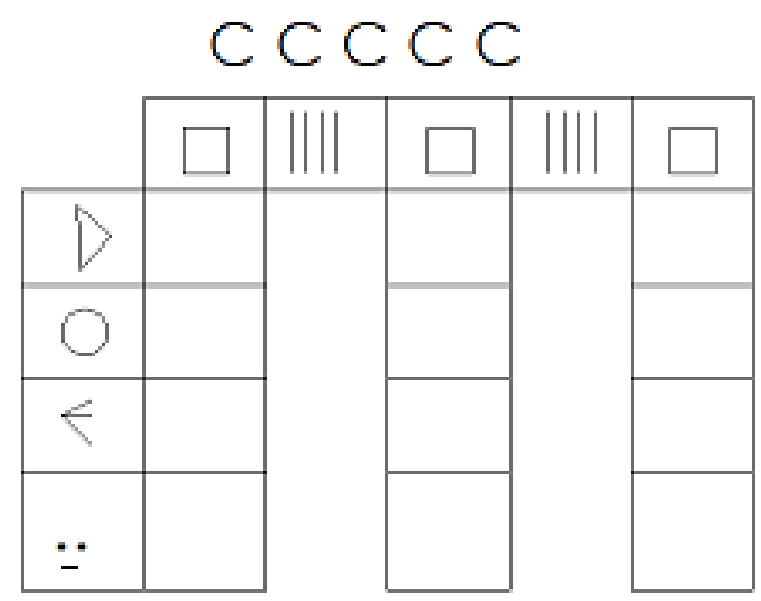

El cuadrado significa "detención" o "transición", las cuatro rayas paralelas "movimiento", el triángulo significa "configuración", el redondel "ubicación", los tres radios "orientación" y, por último, los dos puntos sobre la raya "rasgos no manuales". Quedan así establecidos cinco parámetros de la lengua a través de símbolos y la matriz permite mostrar la forma en que los parámetros configuración, ubicación, orientación y rasgos no manuales constituyen una detención que se articula con otra detención al interior de la seña mediante el parámetro movimiento. A su vez cada parámetro es descripto mediante un código específico (para mayor detalles del modelo cf. BONILLA; PELUSO, 2010).

Todos estos sistemas descriptos anteriormente no son sistemas de escritura, sino de transcripción de las lenguas de señas. Son útiles para lingüistas e investigadores en el campo de los Estudios Sordos y son una importante herramienta, en este momento, en los trabajos de gramatización de las lenguas de señas y, en algunos casos, sirven como instrumento de notación en la producción de textualidad diferida en lengua de señas.

Los dos sistemas de escritura que, como ya señalé, se han creado para aplicarse a las lenguas de señas son: el signwriting y el sistema semasio- 
gráfico de los Testigos de Jehová.

El signwriting es un sistema que se ideó como un sistema de escritura para las lenguas de señas, en el que se escriben todos los parámetros de forma icónica. Tal como se señala en la página oficial de signwriting (http:// signwriting.org), el sistema fue elaborado por Valerie Sutton basándose en un sistema de escritura que ella misma había desarrollado con anterioridad a efectos de escribir los movimientos de danza. En la próxima imagen, extraída de http://en.wikipedia.org/wiki/SignWriting se observa un texto escrito en dicho sistema. La escritura se realiza de arriba hacia abajo y toma en cuenta los siguientes aspectos: orientación; configuración manual; movimiento de los dedos; movimiento de la mano; movimiento de ojos, hombros y cabeza; movimiento de la cara (expresión); movimiento del cuerpo; ubicación; y prosodia (velocidad, intensidad, etc.). También se agregaron signos de puntuación, lo que es clave en la textualidad escrita para marcar estructuras textuales. Si bien es un sistema icónico, por ser sumamente exhaustivo, es un sistema que requiere de muchos símbolos y, por lo tanto, es bastante complejo en su uso.

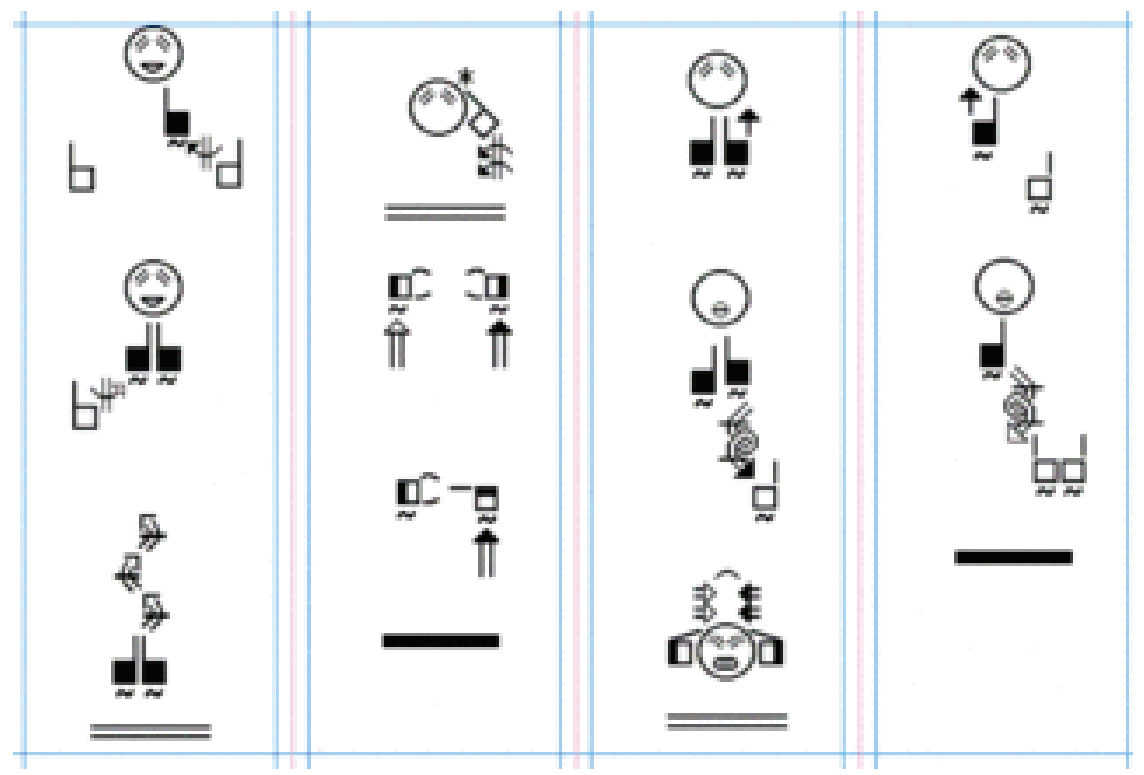

Fonte: http://en.wikipedia.org/wiki/SignWriting 
El signwriting es el único sistema de escritura que ha sido adoptado por algunas comunidades como sistema de escritura y ha sido enseñado en escuelas bilingües como parte de la alfabetización de los niños sordos en su lengua. Se está comenzando a utilizar este sistema de escritura en una Wikipedia en American Sign Language (ASL Wikipedia Project: http://ase. wikipedia.wmflabs.org/wiki/Main_Page ).

No hay evidencia, en investigaciones realizadas, que el signwriting ocupe similar lugar en el procesamiento del lenguaje que los sistemas de escritura de las lenguas orales.

Por último, los equipos de Testigos de Jehová sordos y oyentes que hablan la lengua de señas han desarrollado un sistema semasiográfico, a punto de partida de las varias investigaciones existentes en mapas conceptuales, según el cual pueden representar las ideas específicas (no así las unidades de la lengua) y resulta novedoso la aplicación que ellos hacen a la lengua de señas. Este sistema parece ser particularmente útil en los trabajos de traducción de textos en lengua oral escrita a videograbaciones en lengua de señas.

La metodología que se sigue para la realización de esta escritura semasiográfica es dibujar las ideas específicas y sus relaciones (algo similar a un mapa semántico, o mapa conceptual, pero sin palabras escritas) de acuerdo a dos posibles fuentes, un texto propio que está produciendo quien escribe o a partir de un texto (en lengua oral o en lengua de señas) dicho por otro.

Los escribas que poseen esta metodología de escritura semasiográfica están capacitados para escribir las ideas específicas de acuerdo a su criterio: hay quienes utilizan signos más de tipo matemático y hay quienes prefieren dibujos más icónicos. Pero en todos los casos son dibujos propios, no compartidos por otros ni estandarizados. En general, cada escritor que utiliza este sistema semasiográfico busca estabilizar relaciones entre ideas y dibujos, por lo que a medida que internaliza este sistema los dibujos van recurriendo en cada escriba, en tanto recurren las mismas ideas específicas.

A continuación presento un ejemplo de escritura semasiográfica, la misma fue realizada en una clase de psicología del lenguaje. 
(1)
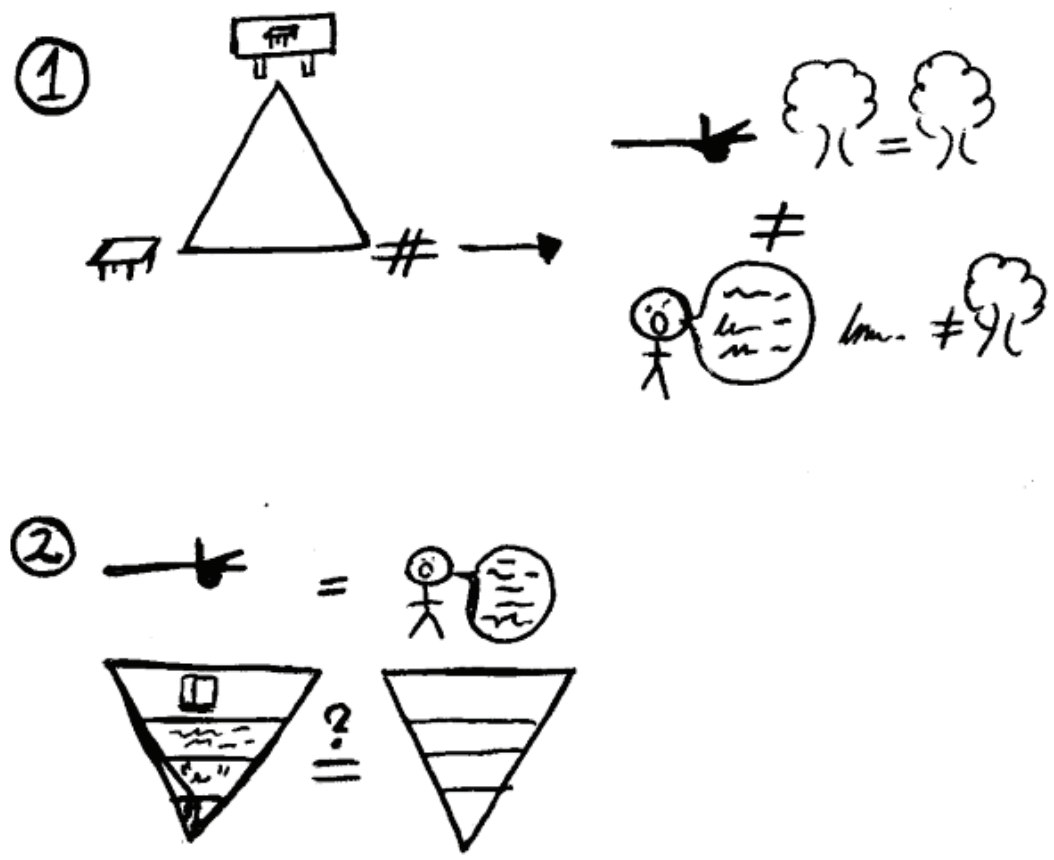

1) el triángulo de Pierce supone que en el extremo inferior izquierdo está el objeto representado, en el extremo superior la representación y en el extremo inferior derecho el código. La lengua de señas se diferencia de la lengua oral en que la primera incluye la iconicidad en el código.

2) La lengua de señas es igual, a nivel computativo, que la lengua oral. Tienen los mismos niveles de análisis. Salvo en que se discute, para el caso de la lengua de señas, la existencia del nivel fonológico, tal como se entiende para el caso de las lenguas orales.

Dado que los dibujos son personales y no son estandarizados, este tipo de escritura es personal y, por lo tanto, no es compartible con el entorno. Es un sistema de escritura que tiene una función internamente orientada, es decir, para el propio uso del escritor. No tiene ninguna función comunicacional. Por esta razón es un sistema también muy dependiente de los recuerdos del propio escritor, que le permitan recuperar la información que no está escrita 
(lo mismo ocurre con todas las producciones intermedias: esquemas, mapas conceptuales en lengua oral, etc.). Esta escritura semasiográfica es muy útil para el caso de la traducción de la lengua oral escrita hacia la lengua de señas diferida (videograbaciones como escritura), dado que permite escribir un guión a quien va a realizar la videograbación que está completamente desprendido de la escritura de la lengua oral y de sus estructuras.

\section{Los sordos como cultura eminentemente ágrafa}

Si caracterizamos a los sistemas de escritura como aquellos sistemas que mediante marcas visibles y permanentes pueden representar las unidades de la lengua, separando así el texto del momento de su enunciación, se puede decir que en Uruguay no existe ningún sistema de escritura para la LSU. Dicha lengua no cuenta con el nivel fonológico: cada seña está compuesta por parámetros y resulta sumamente engorroso transcribir cada parámetro a la hora de escribir cada seña.

Debido a ello los sordos en Uruguay tienen que aprender a escribir en una segunda lengua, en lugar de aprender un sistema para representar las unidades de la propia. Esta situación hace que, en general, nuestra comunidad sorda haya mantenido una fuerte relación de ajenidad con respecto a la escritura.

Por tener la escritura de los sordos un carácter de segunda lengua, frecuentemente éstos presentan dificultades a la hora de leer textos formales (aparentemente esto no sería completamente extensible a los sms y al chat, en donde la sintaxis tiende a ser propia de la oralidad). En cuanto a escribir, en general aparecen, en los textos elaborados por sordos, marcas de hablantes no nativos e interferencias de la morfosintaxis de la LSU. Es importante remarcar que estas marcas no tienen nada que ver con una patología a nivel de la escritura, sino que responden a que la escritura, para los sordos, implica el dominio de una segunda lengua.

Esta situación, bastante extraña para el común de los bilingüismos, supone una extrema distribución funcional entre las lenguas implicadas: en la oralidad se utiliza casi exclusivamente la LSU y en la escritura se usa el español. Una distribución de esta naturaleza trae aparejadas, necesariamente, consecuencias en las características de las lenguas y sus relaciones y en la forma en que los hablantes se las representan. 
Tenemos una lengua largamente estandarizada y acuñada, enmarcada en una ideología letrada en relación con una lengua escasamente estandarizada que carece de forma escrita. Esto actualiza, inevitablemente relaciones de poder y prestigio entre ambas, que afectan a sus hablantes y a la forma en que se ven a sí mismos como hablantes de una u otra lengua. En el caso de los alumnos sordos, por ejemplo, muchos manifestaron en las entrevistas que realicé en una investigación en el Liceo 32 en el año 2001 que el español era una lengua en la que hablaban de cosas importantes, vinculadas a lo científico y extrafamiliar, pero que en LSU hablaban de pavadas, básicamente asuntos de sordos. Estas ideas sobre las lenguas están siendo rápidamente modificadas con los procesos de intelectualización que está experimentando la LSU, si bien persiste la situación de ser una lengua no letrada. Ante esto surge la pregunta: ¿Por qué la LSU tendría que ponerse por escrito? ¿Es una necesidad que surge en la comunidad sorda?

Son varios los motivos por los que una lengua tiene que ponerse por escrito en el contexto globalizado y altamente letrado que viven nuestras culturas occidentales del siglo XXI; motivos que no surgen de la comunidad sorda, sino que se le imponen desde el afuera como forma de dialogar en paralelo con las otras comunidades lingüísticas con las que comparte el mismo espacio de Estado posmoderno.

Para poder ser lengua de la educación, de la literatura, de la ciencia, de las leyes, de registro de identidad y de propiedad, de la religión, la lengua tiene que tener, en nuestras comunidades, su escritura, ya que todas estas funciones requieren de textos estabilizados, que puedan perdurar más allá del momento de enunciación, es decir, que se puedan archivar y, en algunos casos, interpretar (ciencia y literatura).

A su vez la propia estandarización requiere de un sistema de escritura que permita generar diccionarios y gramáticas descriptivas de la lengua a efectos de su fijación. Toda lengua que quiera ser enseñada debe contar con gramáticas y diccionarios y por lo tanto de tener escritura.

\section{Herramientas de registro}

Las herramientas de registro son de muy reciente aparición en la $\mathrm{Hu}-$ manidad.Estas herramientas de registro permiten, entre otras cosas, audiograbar o videograbar textos. Se habilita así una textualidad que cumple con 
determinadas características similares a lo que es una textualidad escrita, pero sin serlo. La escritura es una herramienta que, básicamente, lo que hace es representar las unidades de la lengua; las videograbaciones y audiograbaciones lo que hacen es registrar a un hablante hablando la lengua. Representación y registro son dos actividades que tienen puntos en común, pero también importantes puntos de divergencia, como veremos más adelante.

A través del Programa Textualidad Registrada en LSU (TRELSU) que se lleva adelante entre la Facultad de Psicología en Regional Norte - Salto/ UdelaR, la Tecnicatura Universitaria de Interpretación LSU-español-LSU (TUILSU), Facultad de Humanidades y Ciencias de la Educación - Montevideo, Salto, Tacuarembó/UdelaR y la Escuela N ${ }^{\circ} 116$ para sordos y personas con alteración de lenguaje - Salto/ANEP-CEP, se pretende consolidar una textualidad registrada en LSU. Esto supone la creación de una metodología que permita el uso de las videograbaciones como escritura y el desarrollo de un cuerpo teórico concomitante. Para que las videograbaciones puedan funcionar como escritura se requiere que éstas cuenten con una estructura interna, dado que es un elemento clave que impone la textualidad escrita formal (índices, párrafos, títulos, manejo de fuentes bibliográficas, etc.). Asimismo se necesita de la incorporación de técnicas de vidoegrabación que suponen el manejo adecuado del foco, el fondo, la luz y ciertos códigos vestimentarios; así como también el manejo de programas informáticos para la edición de los videos bajo un criterio que señale las diferentes partes (cambio de fondo si se trata de un título o de un párrafo; numeración de los segmentos según capítulos y segundos de filmación; etc.)

Esta textualidad diferida puede provenir de dos fuentes diferentes:

a. textos originariamente construidos en LSU;

b. textos traducidos de otra lengua.

Para la elaboración de textos vidoegrabados en LSU de forma directa se están promoviendo talleres, en el marco del Programa TRELSU, con sordos en la escuela y con sordos adultos de forma tal que puedan contactar con esta idea de la textualidad organizada, lo que supone la planificación del texto a videograbar bajo las condiciones antes expuestas.

Para el caso de la traducción de textos de lengua oral escrita a la lengua de señas, se está desarrollando una metodología, a punto de partida de los avances en la organización de los textos videograbados que mencionaba anteriormente y de los trabajos realizados por el Centro de Traducción de la 
Asociación de Testigos de Jehová. Dicha Asociación es la única que tiene, en la actualidad, un importante avance en el campo de la traducción de textos escritos en lengua oral a videograbaciones en lengua de señas (Sánchez y Salguero, 2011).Como la Asociación solo traduce textos religiosos, el desafío del equipo de trabajo TRELSU es elaborar una metodología que tenga en cuenta las particulares necesidades que tienen los textos académicos.

La elaboración de textualidad diferida en LSU que recoja ambas fuentes de producción tiene la importancia de crear un acervo textual en LSU, actualmente inexistente, en el que se pueda rescatar, entre otras cosas, aspectos de la cultura sorda (literatura, geografía, historia) y en el que se pueda acceder, en LSU, a los textos que son patrimonio de la cultura global y que actualmente solo existen lengua oral escrita.

\section{Diferencias y similitudes entre escribir, transcribir y registrar}

Los textos escritos y los textos videograbados comparten muchas propiedades, lo que hace posible que, bajo ciertas condiciones de producción, las videograbaciones en lengua de señas puedan tomar las estructuras y funciones que en nuestras culturas letradas son particulares de la escritura.

Existen dos propiedades básicas de los sistemas de escritura, que se coimplican y están en la base de la posibilidad de potenciar las funciones metalingüísticas y metacognitivas que son esenciales en el manejo de la textualdiad escrita: la permanencia y la objetivación (ONG, 1982; GOODY; WATT, 1996; SAMPSON, 1997).

La escritura hace que el texto se vuelva permanente, a diferencia del texto en la oralidad que es, como plantea Ong (1982), un texto evanescente. En la oralidad el texto forma parte del enunciador y va desapareciendo a medida en que se va enunciando. Su permanencia depende de la memoria inmediata de quienes hablan. Obviamente que en la medida en que la conversación discurre es imposible ir reteniendo todos los enunciados. A través de la escritura, que representa las unidades de la lengua y sus enunciados, el texto permanece dado que deja de depender de la memoria de quienes interactúan con este. La permanencia del texto depende del soporte físico en el que esté hecho, de las propiedades de archivo y, también, de la función. Una lista de supermercado puede durar el tiempo de compra, mientras que una obra literaria puede perdurar por siglos o milenios. Una frase de amor 
escrita en la arena de la playa durará lo que la marea permita, en cambio esa misma frase puede durar años estampada en una pared del centro de una cuidad.

En este sentido, las videograbaciones comparten con la escritura esta propiedad de permanencia, dado que el texto videograbado también queda fijado.

La objetivación del texto, en el sentido de su transformación en un objeto físico, es otro de los efectos de la escritura. En la medida en que la escritura representa las diferentes unidades de la lengua mediante marcas visibles (o táctiles en el caso del Braile), el texto escrito pasa a tener consistencia física. Lo que en la oralidad es evanescente e intangible, en la escritura es permanente y manipulable. El texto escrito es un objeto. Se transforma en una cosa exterior al enunciador. La posibilidad de tener el texto permanente y en un soporte que lo vuelve manipulable, hacen del texto un objeto que se puede interpretar, transformar, re-escribir. No ocurre así con lo dicho en el marco de un contexto oral.

Las videograbaciones también tienen objetivan al texto y lo vuelven un objeto manipulable externo al hablante. Sin embargo aquí ya comienzan a aparecer ciertas diferencias, dado que el soporte material es diferente, lo que hace más difícil la manipulación del texto. Probablemente la escritura que se realizaba, en la Antigüedad, sobre piedra tampoco resultaba muy manipulable. Sin embargo, los soportes actuales que utiliza la escritura, luego de siglos de evolución, hacen de los textos escritos objetos fácilmente manipulables. No ocurre así con las videograbaciones, en las que no resulta tan fácil recorrer el texto, hacer anotaciones, cambiar sectores, etc.

Otra característica de la escritura y que es efecto de las dos anteriores, es que el texto se vuelve diferido en relación al momento de enunciación. Las videograbaciones también comparten esta característica. El hecho de tener una herramienta que separa el texto del momento de la enunciación y de los enunciadores, hace que el texto quede diferido de su enunciación. No es posible identificar texto y enunciador de la misma forma en que se lo hace en la oralidad. Esto produce importantes efectos sobre la estructura y función de los textos. En la medida en que el texto se separa del momento de enunciación, mucha de la información que acompaña al texto de manera pragmática debe sintactizarse, y esto le otorga especificidades a la estructura lingüística de los textos diferidos. Por otro lado, las posibilidades de diferirse del momento de enunciación le dan al texto nuevas funciones y 
hacen posible, por ejemplo, el trabajo científico, la legislación, el archivo de información, y todas otras funciones que son típicas de las actuales culturas letradas y globales.

Asimismo, y como efecto de la permanencia y la objetivación, el texto se vuelve interpretable. Como plantea Olson (1998) un texto solo es interpretable si está escrito (y se puede agregar que también es interpretable si está videograbado), de lo contrario está sometido a los avatares de la oralidad, en la que el texto es permanentemente co-construido y re-construido por los participantes de la conversación. Interpretable significa que ya no importa qué quiso decir el enunciador; en la escritura lo que importa es qué quiere decir el texto. Se pierde la cuestión dialógica de entender al otro y, en tanto el texto es un objeto independiente del enunciador, se vuelve, por sí mismo, objeto de interpretación. Inclusive el propio escritor pasa a ser interpretador de su texto, con el que pasa a tener una relación de alienación.

Por último, el sistema de escritura al interanlizarse y volverse plano interno forma parte del propio procesamiento del lenguaje. Esto claramente permite separar transcibir de escribir. Cuando se transcribe un texto, así sea en notación fonética, fonológica u ortográfica, lo que se hace es representar las unidades de un texto ya procesado en la oralidad. En ese sentido, la transcripción es una modalidad que estaría a medio camino entre la escritura y la grabación (registro). No sería por completo escritura porque no fue procesada en un inicio como escritura y no es una grabación porque como resultado se obtiene una representación de los enunciados del texto y sus unidades.

En el caso de las videograbaciones cabe señalar que no existe aún ninguna posibilidad de que esta herramienta pueda internalizarse y que forme parte del procesamiento del lenguaje, como ocurre con la escritura. Es siempre un procesamiento externo a la herramienta, aún cuando se esté procesando un texto videograbado.

Otra propiedad de la escritura es ser un sistema esencialmente representacional (OLSON, 1998). Es un sistema que al funcionar representando las unidades de la lengua (tanto en el plano externo como en el plano interno), se transforma en un sistema esencialmente metalingüístico: hace explícito el funcionamiento del lenguaje para sus usuarios. Un sistema de esta naturaleza, que hace explicito el funcionamiento del lenguaje, es necesariamente un sistema metalingüístico y metarepresentacional. La potenciación de la conciencia sobre el lenguaje produce, a su vez, en las culturas letradas, el 
desarrollo de lo que Olson (1991) denominó metalenguaje oral. Este desarrollo y especialización de un procesamiento metalingüístico se potencia con otra herramienta que se aplica a las lenguas y que también apunta a potenciar los niveles metalingüísticos: la tecnología de gramatización.

Las videograbaciones, por su parte, son una herramienta de registro de los enunciados. El hecho de ser una herramienta que registra los enunciados de la lengua, pero no los representa, produce relaciones diferentes de los hablantes con el texto y con la propia lengua, a las que ocurren con el uso de los sistemas de escritura. Frente a un texto escrito, un lector debe realizar determinados procesos cognitivos para poder decodificarlo. Asimismo, por ser representado, ya implica que dicho procesamiento ocurrirá en el nivel metalingüístico más elevado. En cambio, al tener la lengua registrada, el procesamiento es casi similar al que ocurre en un contexto conversacional. El nivel metalingüístico es mucho menor.

\section{Herramientas de gramatización}

Con posterioridad al desarrollo de la escritura, y como uno de sus efectos, surgen las herramientas de gramatización: las gramáticas y los diccionarios (AUROUX, 1992). Si bien ya existían estas herramientas aplicadas al latín y al griego, recién aparecen en Europa aplicadas a las lenguas romances entre el año 1.100 y 1.500 . Estas tecnologías no tienen como objetivo representar a la lengua (como ocurría con los sistemas de escritura) sino describirla en sus diferentes niveles: los diccionarios apuntan a la descripción del nivel léxico de la lengua (aunque a veces también contengan información fonológica y morfológica) y las gramáticas apuntan a la descripción del nivel morfológico y del nivel sintáctico.

Para Auroux (1992) existen dos tipos diferentes de procesos de gramatización, que hacen a diferentes instrumentos: la exogramatización y la endogramatización. La exogramatización resulta de construir diccionarios y gramáticas de una lengua desde fuera de dicha lengua. Por ejemplo, cuando se hicieron las primeras gramáticas del guaraní o del quechua descriptas en español o latín; o cuando se elaboró el primer Léxico del español que era traductor español/latín. En cambo la endogramatización supone un paso más en la evolución del proceso de gramatización sobre una lengua e implica la construcción de instrumentos desde dentro de la lengua. Aparecen los 
diccionarios monolingües y las gramáticas descriptas en la propia lengua a describir.

El efecto que tiene la aplicación de estas herramientas de gramatización sobre la lengua es la producción de una variedad estándar. En la medida en que la gramática y el léxico aparecen explícitamente descriptos, estos niveles se trabajan y expanden (por ejemplo, se rigidizan los límites de las piezas léxicas) al tiempo que se instituye un modelo explícito que pasa a funcionar como norma lingüística a seguir (se consolida el purismo idiomático).

El proceso de gramatización que ha ocurrido sobre la LSU desde fines de los años ochenta a esta parte ha sido tan escaso que aún estamos lejos de tener una lengua plenamente gramatizada. La primera investigación lingüística de rigor científico que se realizó sobre la LSU es el ya clásico: Lengua de Señas uruguaya. Su Componente Léxico Básico (BEHARES; MONTEGHIRFO; DAVIS, 1987). Dicha investigación fue llevada adelante por un equipo que tenía inserción universitaria y contó con colaboración internacional. El siguiente trabajo que se realizó en la gramatización de la LSU fue un Diccionario de dicha lengua: el Diccionario Bilingüe de Lengua de Señas Uruguaya/Español. El mismo fue elaborado por la Asociación de Sordos e instituciones vinculadas, con poca supervisión lingüística y varios errores tanto teóricos como metodológicos, fundamentalmente por la escasa teoría lingüística que hay detrás. Una primera versión del Diccionario apareció en el año 2005, pero su publicación definitiva fue dos años después (ASUR/CINDE, 2007). Ambos textos son instrumentos de exogramatización, en tanto se realizan desde el español escrito e ilustraciones.

A partir del año 2009, y con el inicio de la Tecnicatura Universitaria en Interpretación LSU-español-LSU en la Facultad de Humanidades y Ciencias de la Educación de la Universidad de la República, se retomaron en Uruguay, a nivel académico, los estudios en lingüística de la LSU, que habían sido abandonados a fines de los ochenta luego de la publicación del Léxico Básico de la LSU. Con el ingreso de investigadores sordos a la Universidad y la formación de los lingüistas en el campo de la lingüística de la lengua de señas, Uruguay retomo otra vez el empuje hacia la continuación del proceso de gramartización.

A partir del año 2010 comienza a desarrollarse el sistema de notación TRELSU (BONILLA; PELUSO, 2010). Este sistema de notación propone una un diseño para la matriz segmental articulatoria y lenguajes específicos 
para describir cada parámetro, lo que es la base de descripción lingüística para lo que sería el primer Diccionario LSU-LSU (un instrumento de endogramatización de dicha lengua: es decir totalmente realizado en LSU sin necesidad del español como la lengua de descripción).

En el año 2011 se publicaron dos artículos que avanzan en la línea de los estudios lingüísticos de la LSU. Uno de ellos (TANCREDI, 2011), trata de un análisis de los rasgos no manuales en el que se propone un sistema descriptivo que no usa el español y que, por lo tanto, tiene el especial interés de poder ser incluido como lenguaje descriptor de un parámetro dentro del modelo del nivel fonológico ya señalado y que se está llevando a cabo en el Programa TRELSU. El otro artículo inaugura los estudios gramaticales en tanto trata el tema de la flexión de número en los sustantivos (FOJO, 2011). Este artículo es de relevancia, dado que hasta el momento solo se contaba con análisis de los niveles fonético-fonológico y léxico de la LSU.

En el año 2012 se publica un trabajo sobre los alófonos en la LSU en el que se incorpora la perspectiva sociolingüística dentro del análisis lingüístico que se venía haciendo hasta ese momento de esta lengua (MUSLERA, 2012). También en dicho año se termina de diseñar el programa informático Léxico TRELSU (que capitaliza la investigación lingüística y de software realizada en el Programa TRELSU) y se publicó un trabajo que presenta teóricamente, describe y hace las veces de manual de uso del Léxico TRELSU (PELUSO; VAL, 2012).

Todos estos trabajos apuntan a la gramatización de la LSU, algunos de exogramatización y otros de endogramatización. Los desarrollos de instrumentos de gramatización de la LSU detienen la tradicional variación de generación a generación y según las regiones de la legua e imponen una variedad nacional. En la medida en que una variedad de la LSU está siendo gramatizada y consecuentemente estandarizada y, por lo tanto, está adquiriendo nuevas formas, funciones y estatus, se producen conflictos que involucran a sus hablantes en el marco de una comunidad que tenía una fuerte creencia (ilusión) de homogeneidad como grupo minoritario.

Es importante, entonces, que en todo este proceso en el que la Universidad de la República está teniendo un rol trascendente, los investigadores sordos y oyentes que participamos del mismo tengamos en cuenta estos aspectos políticos e identitarios implicados, que luego tienen efecto en la comunidad y en los marcos de legitimación de todo el proceso. 


\section{El Léxico Básico: un ejemplo de exogramatización}

El primer instrumento de gramatización fue el Léxico Básico de la LSU. El mismo es un texto que, como su nombre lo indica, y así lo expresan los autores en el Prólogo, pretendía documentar un mínimo de piezas léxicas de dicha lengua, las de uso más frecuente, de cara a las nuevas funciones y estatus que se le imponían a la LSU con su ingreso a la educación formal en el marco de la propuesta de educación bilingüe para los sordos que comenzó en ese mismo año. No se propone, por lo tanto, la descripción de los niveles fonológico, morfológico o sintáctico de la LSU.

Su oportuna aparición en el marco de los procesos de estandarización de la LSU, y el hecho de ser la única obra de gramatización realizada sobre la LSU, hizo del Léxico Básico una obra clave que marcó los procesos de estandarización y gramatización de la LSU hasta mediados de los 2000. Si bien hay que reconocer que tuvo menor incidencia de lo esperable por ciertas cuestiones políticas que rodearon dicho trabajo (llegó a ser censurado en ciertas instituciones sordas de prestigio), igualmente fue el único texto de referencia hasta el año 2005.

En cuanto a los aspectos técnicos de la elaboración del Léxico, cabe señalar que

las descripciones de las señas que allí se realizan siguen los criterios de la lingüística de la lengua de señas, de tradición norteamericana, inaugurada por Stokoe (1960) y luego continuada por Liddell y Johnson (1986). El hecho de que la publicación de la obra clave de Liddell y Johnson se haya realizado solo un año antes que la publicación del Léxico Básico, indica, entre otras cosas, la adecuación científica de esta obra para el momento en que fue escrita y su total vigencia en la actualidad.

La metodología de descripción de cada pieza léxica partió de un breve análisis lingüístico de la LSU que consistió en describir los movimientos y las configuraciones de la mano encontradas en las señas.

Para el caso del movimiento, junto al dibujo del mismo, aparece su descripción en español escrito. Se describen 17 movimientos. 
El círculo muestra el lugar exacto en que comienza la seña.

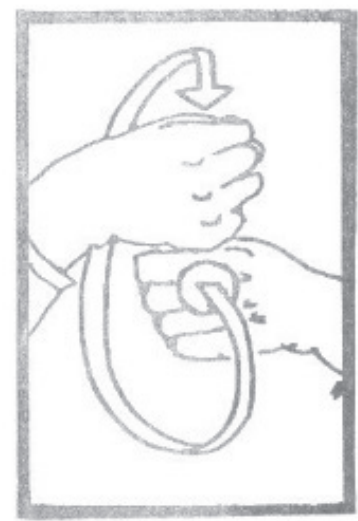

Las configuraciones de la mano aparecen dibujadas.

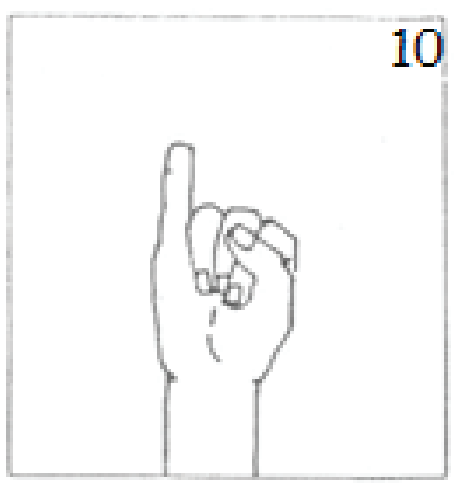

A cada configuración se le asignó un número (el número 10 en este caso ejemplo). En ningún caso se les dio un nombre en español. Se consignaron 53 configuraciones de la mano.

En el Léxico propiamente dicho se describen 325 piezas léxicas, organizadas por dominios de uso (por ejemplo: vestimenta, la casa y sus partes, alimentación, paisaje natural, etc.).

Cada entrada léxica consta de tres partes claramente diferenciadas: 
dibujo; asignación numérica de la seña y de la configuración manual; y el texto en español.
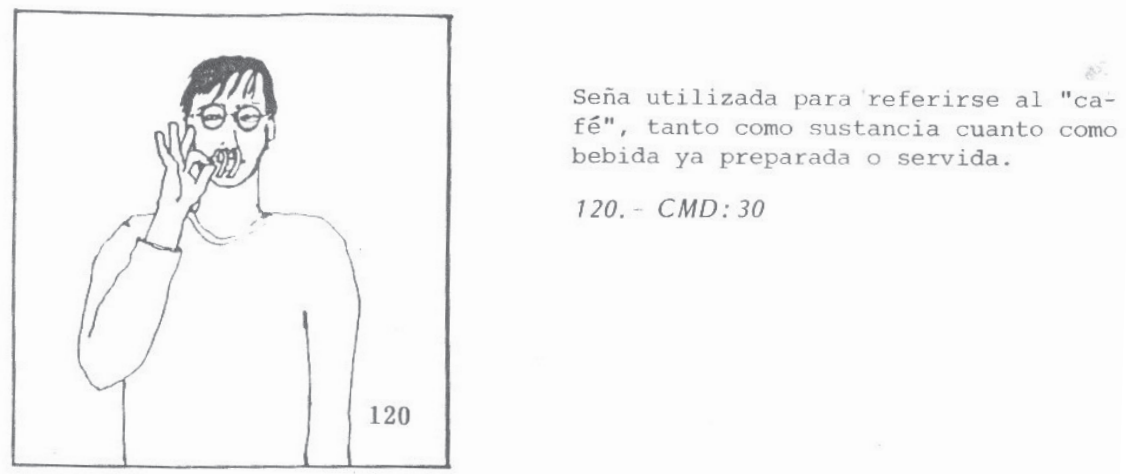

El dibujo se focaliza en los rasgos manuales de la seña: parámetros configuración, orientación, ubicación y movimiento. En los casos en que es lingüísticamente relevante se consignan los rasgos no manuales mediante el propio dibujo, una foto o un comentario en español escrito.

La asignación numérica es doble. Por un lado se dio un número a cada seña según su orden de aparición en el Léxico, que se corresponde a su organización por dominios de uso (en este ejemplo el número 120). Por otro lado se le asigna el número que corresponde a la configuración de la mano dominante (CMD), que en el ejemplo anterior corresponde al número 30. En ambos casos esto tiene como función darle a cada seña descripta criterios de ubicación para ser manejados a través de los índices que están colocados al final.

El texto en español escrito que acompaña los dibujos contiene tres tipos de información: el significado de la seña, un análisis de las variaciones de las señas (se especifican variantes, señas de uso sinónimo, etc.) y la traducción al español.

Al final del texto aparecen los 3 índices de búsqueda del Léxico:

- Índice de acuerdo a la configuración inicial de la mano. 


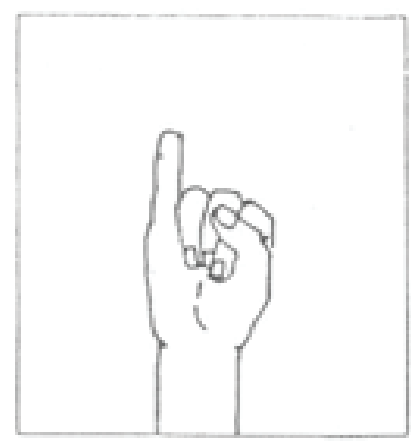

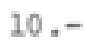

130

177

213

223

265

310 fideo, pasta

dra, de dra

Italia

feliz, contento, alegre

flaco, delgado, fino

si, si

En este caso bajo la égida de cada dibujo que representa una configuración manual (ordenadas por el número de configuración previamente asignado: en el ejemplo que aparece anteriormente con el número 10) se clasificaron todas las señas que tienen dicha configuración como configuración inicial de la mano dominante.

Cada una de estas señas aparece escrita en español (como glosa) y tiene una indicación del número que se le asignó a la seña en el Léxico. Por ejemplo, la seña fideo tiene la configuración 10 y está ubicada en el lugar 130 según su dominio de uso.

Este criterio de búsqueda es muy importante porque permite la búsqueda desde la LSU y no desde el español.

- Índice por dominios de uso, que corresponde a la organización básica del Léxico.

- Índice alfabético de palabras españolas por las cuales se pueden traducir las señas. 

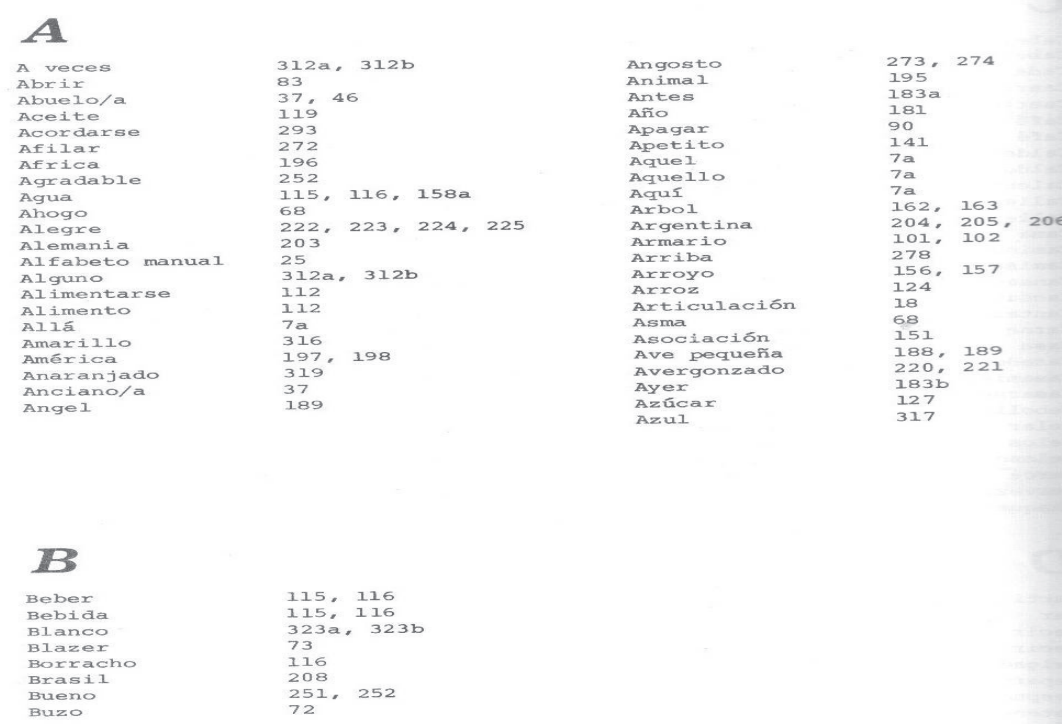

Este índice sigue un orden alfabético. Bajo cada letra se consignan diferentes palabras en español y se les asigna un número, que se corresponde con el número de la seña que dicha palabra traduce. Esto permite la búsqueda de las señas desde el español. Por ejemplo, la seña que traduce la palabra beber corresponde a la número 115 o 116.

\section{EI Léxico TRELSU: primer instrumento de endogramatización de la LSU}

El Léxico TRELSU es un instrumento de endogramatización, dado que se pretende describir la LSU sin utilizar otra lengua que no sea la propia lengua descripta, tal como ocurre en el Léxico Básico. Para ello fue necesario articular los desarrollos en lingüística de descripción del nivel fonológico de la LSU, las videograbaciones de la LSU como textualidad diferida y la informática que permite organizar los diferentes componentes. Esto es necesario dado que al no existir escritura para la LSU, si se pretende elaborar un instrumento de endogramatización de dicha lengua, hay que buscar alternativas tecnológicas a efectos de suplantar la escritura, que ha sido la herramienta central en los procesos de gramatización de las lenguas orales. 
En este diccionario se asocia el video de una seña con el video de su definición (para ello se requiere de toda la tecnología que mostré antes en relación a la elaboración de textualidad diferida en LSU). Para ingresar cada seña y poder luego localizarla entre todas las señas (el equivalente a la búsqueda alfabética en los diccionarios de las lenguas orales), hubo que darle un valor paramétrico a las mismas, que en el caso de este diccionario es exclusivamente a través del parámetro configuración en su valor inicial y final. Al describir este parámetro en las dos detenciones, cada seña queda identificada dentro del conjunto de señas que constituyen el diccionario, y esto habilita a su posterior búsqueda. Esto supuso la elaboración de un programa informático en el que se organizan todos estos datos, lo que habilita la búsqueda paramétrica. Por ejemplo, si quiero buscar la seña SILLA en el diccionario, se deberá otorgar un valor a su configuración inicial (@$>12<+<34>-)$ y un valor a su configuración final $(@->12<"<34>-)$. Al asignar estos dos valores en una tabla de inicio en el programa, este busca y muestra todas las señas que están ingresadas bajo estos valores. El usurario deberá luego elegir de las señas que el programa encuentre, cuál de ellas era la que buscaba.

En el Léxico TRELSU se articulan herramientas lingüísticas de descripción de la lengua, con herramientas de registro (las videograbaciones de las señas y sus definiciones), con tecnologías digitales y con tecnologías comunicacionales. El Léxico va a estar sostenido en una página web, lo que no sólo posibilitará un acceso universal al mismo, sino también posibilidades interactivas de sus usuarios con el instrumento de gramatización.

\section{LSU y tecnologías de información y comunicación}

Las tecnologías que se aplican sobre las lenguas de aparición más tardía son las tecnologías informáticas y digitales. Estas tecnologías, que también se sostienen en parte en los sistemas de escritura y de registro, llevan el procesamiento del lenguaje a otros niveles, en los que se integra, de manera novedosa, la imagen, la escritura y el audio. Así, con estas tecnologías, surgen nuevas funciones de la escritura que la alejan de la variedad estándar (sms, chat), se consolida la función vinculada a los hipertextos, aparece el uso de la internet que impone nuevas estrategias de búsqueda, se revolucionan las posibilidades de reescritura de los textos y de cortado y pegado, lo que 
cambia las estrategias lingüísticas y cognitivas con las que abordamos la escritura de un texto, así como también las posibilidades de plagio (se borran ciertos límites entre lo propio y lo ajeno).

Como ya señalé, la informática juega un rol clave tanto en la edición y organización de los videos como textualidad diferida; como también en la realización del Léxico TRELSU.

Sin embargo, también está teniendo un importante rol en las nuevas estructuras y funciones que está adquiriendo la escritura del español para los sordos. Los sordos participan de chats en español escrito, foros en internet, blogs, búsquedas, sms. Esto ha cambiado la tradicional ajenidad con la que los sordos contactaban con la escritura del español, ya que ahora se ha vuelto una tecnología que se adapta a determinados usos de la oralidad, absolutamente útiles para la comunidad y que han modificado las posibilidades de comunicación, dada la imposibilidad de usar teléfonos tradicionales.

También las tecnologías de la información y la comunicación, con el uso de videocámaras, han habilitado el chat directamente en lengua de señas, modalidad que también se ha extendido enormemente dentro de la comunidad sorda.

\section{Referencias}

ASUR/CINDE. Diccionario Bilingüe de Lengua de Señas Uruguaya/Español. Montevideo: ASUR/CINDE, 2007.

AUROUX, S. A revolução tecnológica da gramatização. Campinas: Editora da Unicamp, 1992.

BARRIOS, L.; BIANCHI, F.; MUSLERA, S.; PELUSO, L.; PIÑEYRO, M. (2003). Apuntes acerca del proceso de creación léxica de la LSU en el Liceo. Separata N.1, Revista del 32, Montevideo, Año III, n. 2, p. 1 - 6.

BEHARES, L. E.; MONTEGHIRFO, N.; DAVIS, D. Lengua de Señas uruguaya. Su Componente Léxico Básico. Montevideo: Instituto Interamericano del Niño, 1987.

BONILLA, F.; PELUSO, L. Hacia un descriptor del nivel fonológico de la LSU. LSI, Lengua de señas e interpretación, Montevideo, n. 1, p. 29 - 56. 2010 
FOJO, A.; MASSONE, M. I. Estructuras lingüísticas de la Lengua de Señas Uruguaya. Montevideo: TUILSU/UDELAR, 2012.

GARVIN, P. L.; MATHIOT, M. La urbanización del idioma guaraní. Problema de lengua y cultura. In: GARVIN, P. L.; LASTRA, Y. (Ed.). Antología de estudios de etnolingüística y sociolingüistica, México: UNAM. p. 303 313. 1974.

GOODY, J.; WATT, I. Las consecuencias de la cultura escrita. In: GOODY, J. (Comp.). Cultura escrita en sociedades tradicionales, Barcelona: Gedisa, p. $39-82.1996$

INSPECCIÓN NACIONAL DE EDUCACIÓN ESPECIAL. Consejo de Educación Primaria. Propuesta para la implementación de la Educación Bilingüe en el Uruguay, Montevideo: Administración Nacional de Educación Pública. Documento oficial. 1987.

LIDDELL, S. K. THINK and BELIEVE: Sequentiality in American Sign Laguage. Language, Washington, v. 60, n. 2, p. $372-399.1984 .<<$ http:// dx.doi.org/10.2307/413645>>

; JOHNSON, R. E. American Sign Language: The phonological base. Sign Language Studies, Washington, v. 65, p. 195 - 277. 1986.

MARTINET, A. Lingüistica sincrónica. Madrid: Gredos. 1966.

MASSONE, M. I. La Lengua de Señas Argentina. Primer diccionario. Buenos Aires: Sopena, 1993

; MACHADO, M. E. La Lengua de Señas Argentina: Gramática y vocabulario bilingüe. Buenos Aires: Edicial. 1994.

MUSLERA, S. Los alófonos y su aparición en la Lengua de Señas Uruguaya. LSI 3, Montevideo, n. 3, p. 5 - 30. 2012.

OLSON, D. La cultura escrita como actividad metalingüística. En TORRANCE, N. (Comp.). Cultura escrita y oralidad. Barcelona: Gedisa, p. 333 - 358. 1991.

. El mundo sobre papel. Barcelona: Gedisa. 1998.

ONG, W. Oralidad y escritura. Tecnologías de la palabra. Buenos Aires: Fondo de cultura económica, 1996. 
OVIEDO, A. Apuntes para una gramática de la Lengua de Señas Colombiana. Cali: INSOR/Universidad del Valle, 2001.

Peluso, L. (2010). Sordos y Oyentes en un liceo común. Investigación e intervención en un contexto intercultural. Montevideo: Psicolibros/UdelaR. . Acerca de los procesos de gramatización de la LSU: descripción y alcances. Montevideo: AUGM/UDELAR, 2011.

.; VAL, S. LÉXICO TRELSU. Primer Léxico de la LSU en LSU. Caracterización, aspectos teórico-metodológicos y manual de uso. LSI 3, Montevideo, n. 3, p. 31 - 49. 2012.

SAMPSON, G. Sistemas de escritura, Barcelona: Gedisa. 1997.

SÁNCHEZ, O.; SALGUERO, E. (2011). El intérprete de LS en el campo religioso: desafíos y logros. In: FOJO, A.; PELUSO, L. (Dir.). CONGRESO DE INVESTIGADORES DE LENGUA DE SEÑAS Y CULTURA SORDA, 2., 2011, Montevideo [Anais eletrônicos...] Montevideo: Facultad de Humanidades y Ciencias de la Educación, 2011. 1 CD-ROM.

TANCREDI, M. Registro y descripción de los rasgos no manuales en la LSU. LSI 2, Montevideo, n. 2, p.119 - 135. 2011.

WIKIPEDIA. A enciclopedia libre. A Wikipedia é un projeto de enciclopedia colectiva universal e multilingüe establecido na Internet sob o principio wiki. Tiene como objetivo fornecer un contenido reutilizable libre, objetivo e verificable, que todos posan editar e mejorar. Disponible en: $<$ http://ase. wikipedia.wmflabs.org/wiki/Main_Page>. Recuperado en: 18 ago. 2013.

WIKIPEDIA. A enciclopedia libre. A Wikipedia é un projeto de enciclopedia colectiva universal e multilingüe establecido na Internet sob o principio wiki. Tiene como objetivo fornecer un contenido reutilizable libre, objetivo e verificable, que todos posan editar e mejorar. Disponible en: $<\mathrm{http}: / /$ en.wikipedia.org/wiki/SignWriting>. Recuperado en: 18 ago. 2013.

Data de registro: $13 / 04 / 2015$

Data de aceite: 26/08/2015 